$\begin{array}{lr}\text { Universitas } & \text { Vol. } 36 \\ \text { Núm. } 76\end{array}$

\title{
Eficacia de un programa de higiene oral individualizado con refuerzo a través de redes sociales en jóvenes universitarios con diagnóstico de gingivitis
}

\author{
Efficacy of an Individualized Prevention Program Including Social Media Support on University Students with \\ Gingivitis
}

Carlos Alberto Serrano Méndez aniversidad Nacional de Colombia, Colombia caserranom@unal.edu.co

DOI: https://doi.org/10.11144/Javeriana.uo36-76.epho Redalyc: http://www.redalyc.org/articulo.oa? $\mathrm{id}=231252657009$

Karen Andrea Avendaño Calderón Universidad Nacional de Colombia, Colombia

Fecha de recepción: 02/05/16 Fecha de aprobación: 12/06/17

\section{Paula Andrea Moreno Caro \\ Universidad Nacional de Colombia, Colombia}

\section{Resumen:}

Antecedentes: Múltiples estudios epidemiológicos han mostrado una alta prevalencia de gingivitis en adolescentes e individuos jóvenes de Colombia. El Tercer Estudio Nacional de Salud Bucodental describió como aproximadamente el 70\% de los jóvenes de 15 a 24 años presentó hemorragia gingival. Propósito: Evaluar la eficacia de un programa de promoción de la higiene oral individualizado, con soporte a través de las redes sociales, en el control de la gingivitis a corto plazo en jóvenes universitarios de Bogotá, Colombia. Métodos: treinta y ocho estudiantes con gingivitis participaron en el programa. Este programa incluía educación personalizada en higiene oral, remoción profesional de placa bacteriana y cálculo, y actividades de soporte difundidas a través de las redes sociales. Se evaluaron parámetros clínicos periodontales de placa bacteriana, hemorragia al sondaje, profundidad de surco y nivel de inserción clínico al inicio del estudio y en un examen de reevaluación cuatro semanas después. Se utilizó la prueba de Wilcoxon. Resultados: Los estudiantes mostraron una significativa reducción del índice de placa de $72,6 \%$ a $29,6 \%$, del porcentaje de hemorragia al sondaje de $36,7 \%$ a $13,8 \%$, y una ligera reducción de la profundidad de surco y ganancia del nivel de inserción clínico. Esto fue concordante con un aumento de la frecuencia de cepillado dental y uso de seda dental. Conclusión: El programa fue eficaz en el tratamiento de la gingivitis; el uso de las redes sociales fue de gran aceptación. Palabras clave: adultos jóvenes, educación en salud oral, gingivitis, higiene oral, prevención primaria, redes sociales. Áreas temáticas: epidemiología oral; prevención y control.

\begin{abstract}
:
Background: Several epidemiology reports have shown high prevalence of gingivitis among adolescents and young individuals in Colombia. The Third Colombian Oral Health Study described how approximately 70\% of individuals $15-24$ years had gingival bleeding. Objective: To evaluate the efficacy of an individualized oral hygiene program that uses social media to treat gingivitis on young university students from Bogotá, Colombia. Methods: Thirty-eight students with gingivitis participated in a program that included: Individualized oral hygiene instruction, professional removal of plaque and calculus and, recall and support on oral hygiene using social media. Evaluated clinical parameters included: plaque accumulation, bleeding on probing, probing depth and clinical attachment levels, these measured at baseline and at a follow-up examination four weeks later. Wilcoxon test was used. Results: Plaque accumulation was significantly reduced from $72.6 \%$ to $29.6 \%$, similarly bleeding on probing was reduced, from $36.7 \%$ to $13.8 \%$, a slight reduction in probing depth and gain in attachment level was observed. Subjects reported an increased frequency for toothbrushing and use of dental floss. Conclusion: The program showed efficacy in the treatment of gingivitis, subjects were highly positive to the use of social media.
\end{abstract}

Keywords: gingivitis, oral health education, oral hygiene, primary prevention, social media, young adult. Thematic fields: oral epidemiology; prevention and control.

Notas de autor:

a Autor de correspondencia. Correo electrónico: caserranom@unal.edu.co 


\section{INTRODUCCIÓN}

Diferentes estudios clínicos han podido documentar cómo el acúmulo de placa bacteriana a través de un periodo aproximado de tres semanas lleva al desarrollo predecible de la gingivitis, y como el reestablecer un adecuado control de placa bacteriana conduce a la resolución del proceso inflamatorio gingival $[1,2,3]$. Estos hallazgos han llevado a que el énfasis de los programas preventivos en salud oral esté centrado en controlar el factor etiológico principal, el cual es la biopelícula bacteriana.

La presencia de inflamación gingival es común en la población colombiana. Según el Tercer Estudio Nacional de Salud Bucodental del año 1998, aproximadamente un 70\% de los jóvenes entre 15 y 24 años presentaba sangrado gingival [4]. Adicionalmente, el Cuarto Estudio Nacional de Salud Bucodental describió cómo un 21,9\% de los jóvenes de 18 años examinados llenaba los criterios para ser catalogados como casos de periodontitis, según el sistema de clasificación del Centro de Control de Enfermedades de Estados Unidos y la Academia Estadounidense de Periodoncia (CDC-AAP) [5,6].

Estudios de larga duración han demostrado que actividades enfocadas en el control de la placa llevan al mantenimiento de la salud dental y periodontal. Un estudio realizado en Suecia, de 30 años de seguimiento, por Axelsson y colaboradores [7] evaluó 257 pacientes de los 375 incluidos inicialmente en el estudio. Estos pacientes recibieron un programa que comprendía una explicación detallada del origen de la enfermedad periodontal, instrucciones en higiene oral que incluyeron cepillado dental e higiene interdental, así como sesiones de profilaxis con revelado de placa y limpieza mecánica profesional. El intervalo de las citas de control se programó según el riesgo percibido para ocurrencia de enfermedad de cada individuo, cada tres, seis o doce meses.

Los resultados mostraron un bajo nivel de pérdida de dientes, entre 0,01 y 0,06 para diferentes grupos de edad. El porcentaje de placa inicial fue de 50-60\%, comparado con el porcentaje de placa final de $<20 \%$. El número de superficies dentales que desarrollaron caries durante el curso del estudio fue de 1,2 a 2,1. Y, al final del estudio, pocos pacientes presentaban índices de necesidad de tratamiento periodontal $\geq 2$.

Otro programa preventivo similar en pacientes adultos con gingivitis fue implementado por tres años en 126 sujetos de Estados Unidos y Suecia por Bogren y colaboradores [8]. El programa incluyó instrucciones en higiene oral, raspaje supragingival, pulido coronal y citas de mantenimiento cada seis meses. Los sujetos en el momento de inclusión en el estudio presentaron un porcentaje de placa bacteriana promedio de 28,5\%, el cual no varió durante el estudio. El porcentaje de hemorragia al sondaje inicial fue de $21,4 \%$ en promedio y disminuyó en un 5,3\% durante el estudio.

El análisis microbiológico de los pacientes incluidos en el estudio de Bogren y colaboradores [8] fue realizado por Teles y colaboradores [9], y demostró una transición hacia una microflora oral compatible con salud periodontal. Los conteos microbianos disminuyeron de valores de mediana iniciales de $27,31 \times 10^{5}$ a valores de mediana finales de $16,12 \times 10^{5}$. Un tercer estudio de carácter preventivo a un año, realizado por Jönsson y colaboradores [10] evaluó el efecto de un programa de educación individualizada en higiene oral comparado con la instrucción tradicional en 113 pacientes afectados por periodontitis crónica de severidad moderada a avanzada.

El programa individualizado se basó en los principios de la teoría comportamental cognitiva de Bandura [11] y buscaba adaptarse según los problemas, capacidades y objetivos de cada persona, con énfasis en las estrategias de cuidado oral que encajaran mejor en las actividades diarias de cada persona. La reducción del índice gingival en el grupo experimental fue 0,27 mayor que en el grupo control. A su vez, la reducción del índice de placa fue 0,16 mayor en el grupo experimental. Un mayor número de pacientes en el grupo experimental reportó realizar el cepillado dental y la higiene interdental de acuerdo con las instrucciones recibidas. Estos resultados de diferentes programas preventivos evidencian que la instrucción y la motivación personalizada en técnicas de higiene oral llevan a una mejoría de la condición periodontal a largo plazo. 
La utilización de las redes sociales por parte de los jóvenes se ha vuelto generalizada. Un reporte realizado en Singapur por Zheng y Cheok [12] describe cómo en el año 2008 solo el 30\% de los jóvenes de aquel país utilizaba las redes sociales, mientras que en el 2011 un 99\% de los jóvenes eran usuarios de estas redes. Los resultados de un estudio similar llevado a cabo en España por Gómez y colaboradores [13] mostraron que un 91,2\% de los jóvenes universitarios encuestados, de edad promedio 21 años, hacía uso de las redes sociales. De forma similar, en España el estudio de Colás y colaboradores [14] encontró que el 64,4\% de los jóvenes andaluces encuestados hacía uso diario de las redes sociales, en tanto que otro $26 \%$ se conectaba varios días a la semana a estas redes.

Los motivos principales para hacer uso de las redes fueron la sociabilidad, en un 50\% de los casos, y la razón más común el buscar compartir experiencias con los amigos; y después lo psicológico, en el 25\% de los casos, en el marco de la búsqueda de signos de simpatía entre amigos y conocidos. Aunque principalmente utilizadas por motivos sociales o psicológicos, las redes sociales han sido también parte de procesos de docencia y promoción de la salud. En educación, pueden fomentar el aprendizaje colaborativo; la socialización es el factor fundamental del desarrollo de la web 2,0. Se busca incentivar el interés de los usuarios de la red social por leer, observar y comentar acerca de los contenidos subidos a internet, como podría ser la motivación e instrucción en una adecuada higiene oral, así como recordatorios para realizarla de forma constante.

Para fomentar la socialización a través de las redes sociales se pueden establecer foros de discusión, opiniones en el muro asincrónico, usar el chat, subir contenido de artículos o videos relacionados con el tema de aprendizaje o realizar eventos [15]. En el área de la odontopediatría se reportan experiencias sobre el uso de las redes sociales para dar soporte a los padres de niños entre 5 y 9 años acerca de una higiene oral adecuada. Las familias asignadas al grupo experimental, el cual hacía uso de un programa de soporte por internet de tres semanas que incluía caricaturas explicativas, objetivos personalizados, guía acerca del uso de recompensas y seguimiento, reportaron una frecuencia 38\% mayor en la realización del cepillado dental de los niños, en comparación con el grupo control [16]. Sin embargo, no son frecuentes los reportes en la literatura científica sobre el uso de las redes sociales para incentivar un adecuado control de la biopelícula bacteriana.

Sería relevante, considerando el gran impacto que han tenido las redes sociales en los individuos jóvenes, así como su inicial utilización en el campo de la educación en salud oral, investigar cuál es el efecto de un programa preventivo que utilice estas redes en la condición periodontal, incluyendo acúmulo de placa bacteriana y sangrado gingival. Esto, como un primer paso al establecimiento de un programa de larga duración. El objetivo del presente estudio es evaluar la eficacia de este programa de educación de la higiene oral individualizado, con soporte a través del uso de las redes sociales, en el control de la inflamación gingival en jóvenes universitarios de la ciudad de Bogotá, Colombia.

\section{MATERIALES Y MÉTODOS}

\section{Tipo de estudio y muestra de pacientes}

En este estudio descriptivo, con un seguimiento a cuatro semanas, se evaluó el efecto a corto plazo de un programa que pretendía tratar la presencia de gingivitis en un grupo de 38 jóvenes estudiantes universitarios mediante instrucciones en higiene oral individualizadas y refuerzo a través de redes sociales. Durante los meses de enero y febrero del año 2015 se realizó una convocatoria pública en el campus de la sede Bogotá de la Universidad Nacional de Colombia, a través de afiches y folletos informativos, buscando estudiantes interesados en participar en el programa Salud Oral en la U, quienes fueron sometidos a una entrevista y un examen clínico preliminar para verificar el cumplimiento de los criterios de inclusión y exclusión.

Los criterios de inclusión fueron: ser jóvenes universitarios de programas académicos de pregrado con edad $\geq 16$ años, presencia de inflamación gingival — definida como la presencia de un porcentaje de hemorragia al 
sondaje $\geq 20 \%$ - presencia de placa bacteriana con un porcentaje de placa de O'Leary $\geq 20 \%$ [17], y estar dispuesto a participar en el programa de promoción de la higiene oral, con una duración aproximada de dos meses.

Los criterios de exclusión fueron: estudiantes con presencia de periodontitis crónica moderada o avanzada según los criterios CDC-AAP [6], presencia de aparatología de ortodoncia fija, estudiantes en estado de embarazo o en periodo de lactancia, estudiantes con enfermedades sistémicas no controladas o que reciban medicamentos que puedan afectar el estado periodontal, o condiciones que requieran la administración profiláctica de antibióticos para poder realizar el examen periodontal.

El estudio recibió la aprobación del Comité de Ética de la Investigación de la Facultad de Odontología de la Universidad Nacional de Colombia, a través del acta 18-14 del 10 de noviembre de 2014. Todos los estudiantes firmaron un formato de consentimiento informado donde se explicó la naturaleza, los riesgos y los beneficios relacionados con la participación en el proyecto. Según la Resolución 008430 de 1993 del Ministerio de Salud de Colombia, en su artículo 11, el presente estudio es catalogado como de riesgo mínimo, debido a que es un estudio prospectivo en el cual se emplean procedimientos clínicos comunes.

\section{Datos demográficos, hábitos de higiene y examen clínico}

Los datos demográficos recopilados incluyeron: edad, sexo, ciudad de procedencia, programa académico que se cursa y presencia o no del hábito de fumar. Se registró el tiempo desde la última visita al odontólogo, en cuatro intervalos de tiempo: en los últimos seis meses, en el último año, en los últimos dos años o hace más de dos años, así como la frecuencia diaria de cepillado dental y del uso de seda dental.

El examen periodontal de los pacientes se realizó mediante el uso de una sonda periodontal de Carolina del Norte, la cual posee una punta activa de $15 \mathrm{~mm}$ de longitud y con marcas cada milímetro (15 UNC, Hu-Friedy Mfg Co., Chicago, IL, USA). Las mediciones periodontales fueron tomadas por dos de los investigadores (KA y PM), en seis superficies de cada diente, con excepción del índice de placa de O'Leary, que fue valorado en cuatro superficies, y sin tomar medidas en los terceros molares: mesovestibular, centrovestibular, distovestibular, mesolingual, centrolingual y distolingual. Se evaluaron los siguientes parámetros clínicos:

- Índice de placa de O'Leary [17]: medido como la presencia o ausencia de placa bacteriana después de la utilización de solución reveladora de placa. Se evaluaron cuatro superficies por diente (distal, vestibular, mesial y palatino-lingual).

- Porcentaje de hemorragia al sondaje [18]: definida como el porcentaje de superficies que presentaron sangrado cuando se realizó sondaje periodontal hasta el fondo del surco, en un periodo de 10 segundos.

- Margen gingival (MG): distancia en milímetros desde el borde de la encía libre hasta la línea amelocementaria. En caso de presentarse recesiones gingivales toma un valor negativo.

- Profundidad del surco gingival (PB): distancia en milímetros desde el borde la encía libre hasta el fondo del surco o bolsa al momento del sondaje periodontal.

- Nivel de inserción clínico (NIC): se calculará utilizando los valores de profundidad de surco y de margen gingival, de acuerdo con la fórmula $\mathrm{NIC}=-\mathrm{PB} \pm \mathrm{MG}$.

\section{Programa Salud Oral en la U}

Este programa fue una propuesta terapéutica integral en periodoncia, conformada por tres etapas. La primera de ellas incluyó educación en higiene oral y prevención de la gingivitis. Se realizó a través de charlas cortas en las cuales se identificaba la presencia de signos clínicos de patología gingival en los pacientes. Esta 
educación estuvo complementada con contenidos subidos a una página creada en Facebook ${ }^{\circledR}$ con el nombre del programa, con frases, imágenes y videos acerca de la salud oral, la gingivitis y el control de la placa bacteriana. Cada cinco días se subía nuevo contenido. También se creó un grupo de WhatsApp para el envío de mensajes recordatorios de realizar la higiene oral.

La segunda etapa comprendió instrucción personalizada en técnicas de higiene oral. Se suministraron instrucciones sobre el cepillado dental y el uso de la seda dental de forma presencial, tratando de personalizarlas de acuerdo con las necesidades y las dificultades de cada paciente. La técnica de cepillado utilizada fue la de Bass modificado [19], con una frecuencia recomendada de tres veces al día, usando una crema dental con flúor (crema dental Colgate, Colgate-Palmolive S. A., Región Andina, Cali, Colombia). Los pacientes recibieron instrucción en el uso de seda dental con una frecuencia recomendada de una vez al día (hilo dental Colgate, Colgate-Palmolive S. A., Región Andina, Cali, Colombia). Los pacientes recibieron los elementos de higiene oral durante todo el curso del programa. Se enviaron recordatorios periódicos acerca de la realización de la higiene oral a través de Facebook ${ }^{\circ}$ y WhatsApp ${ }^{\circ}$.

En la última etapa se llevó a cabo la remoción profesional de cálculo y placa bacteriana, se realizó raspaje supragingival y, de ser necesario, subgingival, según las características clínicas de cada paciente, para la completa eliminación de todos los irritantes locales.

Las fases de educación en higiene oral y remoción profesional de cálculo y placa bacteriana se desarrollaron durante cuatro citas presenciales. Los pacientes recibieron un examen clínico de reevaluación periodontal después de cuatro semanas de terminar el tratamiento profesional.

\section{Análisis estadístico}

Se calcularon medidas de tendencia central: promedios y medianas, y de dispersión: desviación estándar y rangos, para los parámetros clínicos. Se utilizó la prueba paramétrica test-t para el análisis de las diferencias en los parámetros clínicos antes y después del programa de higiene oral. Los resultados fueron analizados a través del programa $\mathrm{R}$, un programa estadístico colaborativo de distribución gratuita ( $\mathrm{R}$ Development Core Team, Foundation for Statistical Computing, Viena, Austria, ISBN 3-900051-07-0). Las diferencias se consideraron estadísticamente significativas cuando el valor $\mathrm{p}$ fue $<0,05$.

\section{RESULTADOS}

\section{Descripción de la muestra de pacientes}

En el estudio se incluyeron 38 pacientes, con un promedio de edad de 21,1 años (DE 1,9). Participaron 17 mujeres (44,7\%) y 21 hombres (55,2\%). La mayoría de los estudiantes provenía de la ciudad de Bogotá, es decir, 24 de ellos $(63,1 \%)$. La carrera universitaria a la que pertenecían con mayor frecuencia fue Ingeniería (doce estudiantes, 31,5\%), seguida de Medicina (ocho estudiantes, 21,0\%). Solo participó un estudiante de Odontología. Y ninguno de los estudiantes reportó ser fumador.

La mitad de los estudiantes $(\mathrm{n}=19)$ reportó haber asistido al odontólogo en los últimos seis meses, otros 15 habían asistido en el último año, por lo cual cerca del 90\% de los participantes había visitado al odontólogo recientemente. La mayor parte de los estudiantes (24, equivalentes al 63,2\%) reportó cepillarse dos veces al día; sin embargo, solo 10 de ellos (26,3\%) dijeron usar seda dental a diario. 


\section{Efecto del programa de higiene oral}

Durante el examen inicial se evidenció que los estudiantes presentaron un abundante acúmulo de placa bacteriana, el promedio del índice de placa de $\mathrm{O}^{\prime}$ Leary fue de 72,6\% (DE 1,6). De forma similar, el promedio de hemorragia al sondaje fue alto: $36,7 \%$ (DE 1,8). Sin embargo, no presentaron bolsas periodontales. El promedio inicial de profundidad al sondaje fue de $2,2 \mathrm{~mm}$ (DE 0,4), con un rango entre los cuartiles de 2,0 a 2,4 mm, y los valores de pérdida de NIC fueron bajos: $0,19 \mathrm{~mm}$ (DE 0,1).

El programa de higiene oral condujo a una reducción significativa del índice de placa de O 'Leary y del porcentaje de hemorragia al sondaje. El valor final del índice de placa fue de 29,6\% (DE 0,13), p = 2,2 x 10 ${ }^{-16}$, y el porcentaje de hemorragia al sondaje fue $13,8 \%(0,10), p=8,6 \times 10^{-13}$. Los valores de PB y de NIC tuvieron una ligera variación, con una mínima reducción de la profundidad del surco y ganancia de NIC, aunque con significancia estadística para la reducción de PB (tabla 1).

TABLA 1

Efecto clínico del programa de higiene oral individualizado. Promedio (DE)

\begin{tabular}{ccccc}
\hline & Inicio & Reevaluación & Reducción & Valor $p$ \\
\hline \% Indice de placa O'Leary & $72,6(1,6)$ & $29,6(1,3)$ & $43,0(1,5)$ & $2,2 \times 10^{-16} *$ \\
\% Hemorragia al sondaje & $36,7(1,8)$ & $13,2(1,0)$ & $22,9(1,3)$ & $8,6 \times 10^{-13} *$ \\
PB (mm) & $2,2(0,4)$ & $2,1(0,3)$ & $0,1(0,2)$ & $9,3 \times 10^{-5} *$ \\
\hline NIC (mm) & $0,19(0,1)$ & $0,17(0,1)$ & $0,02(0,1)$ & 0,29 \\
\hline \multicolumn{4}{c}{} \\
& *Estadísticamente significativo, p < 0,05. \\
\multicolumn{4}{c}{ DE Desviación estándar }
\end{tabular}

Estos resultados clínicos se vieron acompañados de un aumento considerable en la frecuencia de realización de las actividades de higiene oral (tabla 2).

TABLA 2.

Frecuencia reportada de prácticas de higiene oral, n (\%)

\begin{tabular}{|c|c|c|c|c|c|}
\hline \multicolumn{3}{|c|}{ Cepillado dental } & \multicolumn{3}{|c|}{ Seda dental } \\
\hline Cepillado & Inicial & Reevaluación & & Inicial & Reevaluación \\
\hline 1 vez al día & $7(18,4)$ & $1(2,6)$ & SÍ & $10(26,3)$ & $32(84,2)$ \\
\hline 2 veces al dia & $24(63,2)$ & $6(15,8)$ & $\mathrm{NO}$ & $28(73,7)$ & $6(15.8)$ \\
\hline$\geq 3$ veces al dia & $7(18,4)$ & $31(81,6)$ & & & \\
\hline
\end{tabular}

\section{DISCUSIÓN}

El Tercer Estudio de Salud Bucodental, realizado en Colombia en 1998, encontró que, aproximadamente, el $70 \%$ de la muestra de jóvenes entre los 20 y 24 años de presentaba sangrado gingival [4]. Estos resultados son compatibles con análisis de muestras de jóvenes universitarios de Bogotá, donde se ha encontrado un índice de placa de O'Leary de 56\% y un porcentaje de hemorragia al sondaje de 37\% [20]. Los valores del índice de placa de los estudiantes incluidos en el presente estudio son aún mayores $(72,6 \%)$ y los de hemorragia al sondaje son similares a los descritos (36,7\%). Sin embargo, los estudiantes no presentaron profundidades incrementadas al sondaje, ni una magnitud significativa de pérdida del nivel de inserción clínico, por lo cual el diagnóstico periodontal de ellos sería gingivitis.

Algunas de las características principales de las lesiones gingivales asociadas con placa bacteriana son el hecho de no producir pérdida de la inserción periodontal y el ser reversibles cuando se remueve el agente etiológico bacteriano [21]. Cuando los signos clínicos de gingivitis no son resueltos a través del tratamiento 
profesional y el autocuidado con higiene oral, puede llegar a producirse la destrucción del aparato de inserción periodontal.

En Noruega una serie de estudios con 26 años de seguimiento y que incluyó a 223 individuos, demostró que la presencia constante de signos de inflamación gingival lleva a una mayor pérdida del nivel de inserción clínico. Mientras que las superficies periodontales donde no había inflamación (índice gingival =0) presentaron una pérdida de inserción promedio de $1,94 \mathrm{~mm}$, las que mostraron sangrado (índice gingival =2) tuvieron una pérdida de 3,31 mm [22]. Estos hallazgos resaltan la importancia del control de la inflamación gingival a través de una óptima higiene oral, lo cual incluye lograr la cooperación del paciente y brindarle un adecuado conocimiento acerca del proceso patogénico de la enfermedad.

Para poder modificar patrones de comportamiento establecidos es necesario que los profesionales ofrezcan soporte a los pacientes a largo plazo [23]. El presente programa de higiene oral pretendió proveer a los pacientes con un adecuado conocimiento acerca de las técnicas de higiene oral, así como darles soporte permanente en sus esfuerzos con la ayuda de las redes sociales. Los resultados del programa fueron muy positivos, los pacientes permanecieron adheridos a este durante el periodo inicial y los valores tanto de índice de placa como de porcentaje de hemorragia al sondaje se redujeron a valores de menos de la mitad de los valores iniciales. Al ser este estudio la primera aplicación clínica del programa de higiene con refuerzo a través de las redes sociales, no se realizó una comparación directa con un grupo que recibiera la instrucción en higiene oral usual sin el uso de estas redes.

Resulta interesante que si bien 34 de los 38 participantes reportaron haber asistido el odontólogo en el último año para recibir cuidado oral convencional, esto no se reflejó en buen control de placa y baja inflamación gingival, lo cual puede dar soporte al presente tipo de programa preventivo. Por otro lado, hay reportes en la literatura de cómo la realización de ejercicios de higiene oral dentro de estudios clínicos en ambientes controlados puede llevar a la obtención de una reducción de valores de índice de placa de aproximadamente el 42\%, similares a los del presente estudio [24]. El uso de las redes sociales podría ser comparado con otros mecanismos de apoyo, o con la no realización de estos en un próximo paso.

El estudio clásico de Axelsson y colaboradores [7], con un seguimiento a 30 años, demostró que un programa basado en enseñar a los pacientes los signos de la inflamación gingival y realizar un autocuidado diario, a través de la higiene oral, era efectivo en obtener salud dental y periodontal. Los valores iniciales del índice de placa fueron de 50-60\%, mientras que los finales fueron $<20 \%$, hallazgos similares al presente estudio. En un comienzo, 27\% de las superficies dentales examinadas presentaron sangrado gingival, cálculos o bolsas, mientras que al final del estudio solo el 1\% de las superficies presentó algunas de estas características. En el presente estudio el sangrado gingival también fue drásticamente reducido.

Un desarrollo reciente, incorporado a los programas preventivos establecidos, ha sido la propuesta de un programa individualizado descrito por Jönsson y colaboradores [10], el cual pretende considerar los problemas, los objetivos y las capacidades de cada persona dentro de las actividades terapéuticas en siete etapas: 1) análisis de expectativas, conocimiento y motivación, 2) análisis de comportamientos en higiene oral, 3) destreza en el uso de herramientas para la higiene oral, 4) objetivos individualizados de higiene oral, 5.) automonitoreo continuo, 6) incorporación del comportamiento en la vida diaria y 7) prevención de la recidiva.

Los resultados del estudio mostraron una reducción del índice gingival de un valor inicial de 0,92 a 0,21 un año después en el grupo experimental, y de 0,92 a 0,52 en el grupo control. La diferencia fue estadísticamente significativa a favor del grupo experimental. Los resultados del presente estudio muestran una reducción significativa similar de la inflamación gingival, aunque medida a través de un parámetro clínico diferente; el uso de las redes sociales podría ser de utilidad en los pasos relacionados con el automonitoreo, la incorporación a la vida diaria y la prevención de la recidiva descritos por Jönsson y colaboradores [10].

En la actualidad, la utilización del internet y de las redes sociales por parte de los jóvenes es muy frecuente [12-14]. Esta tendencia ha llevado a que en la literatura científica comiencen a aparecer estudios que evalúan 
el uso de internet con el objetivo de proveer conocimientos acerca de los hábitos adecuados de salud oral. Sin embargo, usar internet para buscar información sobre qué es una adecuada salud bucal y las técnicas de higiene oral, no parece ser, precisamente, una práctica muy extendida. Estudios realizados en Gales e India muestran cómo solo $2,7 \%$ y $26,2 \%$ de los pacientes o padres de niños que estaban recibiendo tratamiento odontológico utilizaron internet para obtener información sobre salud oral [25,26].

No obstante, los cambios rápidos que están sucediendo en la frecuencia del uso de estas tecnologías pueden llevar a que estas herramientas sean cada vez más utilizadas en la promoción y prevención de la salud oral, en el presente estudio los 38 estudiantes evaluados permanecieron a corto plazo vinculados activamente a través del uso de las redes sociales.

Otras referencias en la literatura han incluido internet dentro de programas preventivos. Así, el estudio de Gibbs y colaboradores [27] evaluó el efecto de un programa preventivo dirigido a padres de niños de uno a cuatro años, provenientes de familias de inmigrantes en Melbourne, Australia, comparado con una intervención control. El estudio incluyó dos sesiones de tres horas sobre salud e higiene oral, una visita a la clínica odontológica más cercana al lugar de residencia de las familias y recordatorios a través de mensajes de texto, correos electrónicos o correo físico, durante cuatro meses. Los resultados mostraron un riesgo relativo menor para acúmulo de placa bacteriana $(\mathrm{OR}=0,44)$ e inflamación gingival $(\mathrm{OR}=0,34)$ en los niños asignados al grupo experimental. Sin embargo, el $47 \%$ de las 521 familias inicialmente incluidas en el estudio no terminó el estudio.

El estudio de Hurling y colaboradores [16] evaluó el uso de un programa de soporte por internet con el objetivo de mejorar la higiene oral de niños de cinco a nueve años. El contenido ofrecido por internet incluía: caricaturas explicativas, objetivos personalizados y guías acerca del uso de recompensas y seguimiento. Los niños que recibieron el programa de soporte reportaron una frecuencia 38\% mayor de realización del cepillado dental.

El programa Salud Oral en la U ofrece un paso inicial hacia el uso de internet para mejorar hábitos de higiene oral de jóvenes universitarios, el cual puede incluir material educativo y de soporte diverso. En un siguiente paso, el efecto del programa debe ser comparado con una intervención control a un mayor tiempo de seguimiento.

\section{CONCLUSIONES}

Un programa de promoción de la higiene oral individualizado con el uso activo de las redes sociales demostró ser eficaz en el tratamiento de la gingivitis en jóvenes universitarios de la ciudad de Bogotá, en el corto plazo.

\section{RECOMENDACIONES}

Esta estrategia debe ser evaluada en un plazo mayor y comparada con otras estrategias convencionales de instrucción en higiene oral que no incluyan la utilización de redes sociales para brindar soporte a los pacientes.

\section{REFERENCIAS}

1. Loe H, Theilade E, Borglum JS. Experimental gingivitis in man. J Periodontol. 1965 Jan; 35(1): 5-15.

2. Trombelli L, Tatakis DN, Scapoli C, Bottega S, Orlandini E, Tosi M. Modulation of clinical expression of plaqueinduced gingivitis. II. Identification of "high-responder" and "low-responder" subjects. J Clin Periodontol. 2004 Apr; 31(4): 239-52.

3. Abbas F, van der Velden U, Hart A, Moorer WR, Vroom TM, Scholte G. Bleeding/plaque ratio and the development of gingival inflammation. J Clin Periodontol. 1986 Sep; 13(9): 774-82. 
4. Franco AM, Suarez E, Tovar S, Jácome S, Ruiz JA. III Estudio Nacional de Salud Bucal: Enfermedad Periodontal. Bogotá, Colombia: Ministerio de Salud y Protección Social; 1999. pp. 99-108.

5. Peñaloza RE, Suárez E, Palacio Y, Garnica JA, Gómez LC, Sánchez H, Becerra A, Uzaheta A, Amaya J, Escobar D. IV Estudio Nacional de Salud Bucal: Estado Periodontal. Bogotá, Colombia: Ministerio de Salud y Protección Social; 2015. pp. 77-85.

6. Page R, Eke PI, Wei L, Thorton-Evans GO, Genco RJ. Update of the case definitions for population-based surveillance of periodontitis. J Periodontol. 2012 Dec; 83(12): 1149-54.

7. Axelsson P, Nystrom B, Lindhe J. The long-term effect of a plaque control program on tooth mortality, caries and periodontal disease in adults. J Clin Periodontol. 2004 Sep; 31(9): 749-57.

8. Bogren A, Teles R, Torresyap G, Haffajee AD, Socransky SS, Lindhe J, Wennstrom JL. A three-year prospective study of adults with gingivitis. I. Clinical periodontal parameters. J Clin Periodontol. 2007 Jan; 34(1): 1-6.

9. Teles RP, Bogren A, Patel M, Wennstrom JL, Socransky SS, Haffajee AD. A three-year prospective study of adult subjects with gingivitis II: Microbiological parameters. J Clin Periodontol. 2007 Jan; 34(1): 7-17.

10. Jönnsson B, Ohm K, Öscarsson N, Lundberg P. The effectiveness of an individually tailored oral hygiene educational program on oral hygiene behavior in patients with periodontal disease: A blinded randomized controlled clinical trial. One-year follow-up. J Clin Periodontol. 2009 Dec; 38(12): 1025-34.

11. Bandura A. Self-efficacy towards a unifying theory of behavior change. Psychol Rev. 1977 Mar; 84(2): 191-215.

12. Zheng R, Cheok A. Singaporean adolescent perception on online social communication: an exploratory factor analysis. J Educ Comput Res. 2011 Mar; 45(2): 203-21.

13. Gómez M, Rosas S, Farías P. El uso académico de las redes sociales. Comunicar: Rev Cientif Comunic Educ. 2012 Mar; 19(38): 131-8.

14. Colás P, González T, de Pablos J. Juventud y redes sociales: motivaciones y usos preferentes. Comunicar: Rev Cientif Comunic Educ. 2013 Mar; 20(40): 15-23.

15. García-Sans A. Las redes sociales como herramientas para el aprendizaje colectivo: una experiencia con Facebook. Periodismo Comunic Soc.. 2008 Jul; 2(5): 49-59.

16. Hurling R, Claessen JP, Nicholson J, Schafer F, Tomiln CC, Lowe CF. Automated coaching to help parents increase their children's brushing frequency: An exploratory trial. Community Dent Health. 2013 Jun; 30(2): 88-93.

17. O’Leary TJ, Drake RB, Naylor JE. The plaque control record. J Periodontol. 1972 Jan; 43(1): 38.

18. Joss A, Adler R, Lang NP. Bleeding on probing. A parameter for monitoring periodontal conditions in clinical practice. J Clin Periodontol. 1994 Jul; 21(6): 402-8.

19. Bass CC. An effective method of personal oral hygiene. J Louisiana Med Soc. 1954 Mar; (106): 100-12.

20. Marulanda AM, Coral D, Sabogal D, Serrano C. Periodontal conditions of Colombian university students aged 16 to 35. Braz Oral Res. 2014 Jan; 28(1): 1-7.

21. Mariotti A. Dental plaque-induced gingival diseases. Ann Periodontol. 1999 Dec; 4(1): 7-19.

22. Schatzle M, Löe H, Burgin W, Anerud A, Boysen H, Lang NP. Clinical Course of chronic periodontitis. I. Role of gingivitis. J Clin Periodontol. 2003 Oct; 30(10): 887-901.

23. Westfelt E. Rationale of mechanical plaque control. J Clin Periodontol. 1996 Mar; 23(3): 263-7.

24. Chapple ILC, van der Wejden F, Doerfer C, Herrera D, Shapira L, Polak D, Madianos P, Louropoulos A, Machtei E, Domos N, Greenwell H, van Winkelhoff AJ, Kuru BE, Arweiler N, Teughels W, Aimetti M, Molina A, Montero E, Graziani F. Primary prevention of periodontitis: Managing gingivitis. J Clin Periodontol. 2015 Jun; 42 (Suppl. 16): S71-S76.

25. Harris CE, Chestnut IG. The use of the internet to access oral health-related information by patients attending dental hygiene clinics. Int J Dent Hyg. 2005 May; 3(2): 70-3.

26. Naganandini S, Rhao R, Kulkami SB. Survey on the use of the internet as a source of oral health information among dental patients in Bangalore, India. Oral Health Prev Dent. 2014 Mar; 12(2): 141-7.

27. Gibbs L, Waters E, Christian B. Teeth tales: A community-based child oral health promotion trial with migrant families in Austalia. BMJ Open 2015. Jun; 11(5): 6. 


\section{Licencia Creative Commons CC BY 4.0}

Cómo citar este artículo: Serrano CA, Avendaño KA, Moreno PA. Eficacia de un programa de higiene oral individualizado con refuerzo a través de redes sociales en jóvenes universitarios con diagnóstico de gingivitis. Univ Odontol. 2017 Ene-Jun; 36(76): 1-10. https://doi.org/10.11144/Javeriana.uo36-76.epho 